\title{
A Scalable, Out-of-Band Diagnostics Architecture for International Space Station Systems Support
}

\author{
Daryl P. Fletcher \\ Science Applications International Corporation (SAIC) \\ NASA Ames Research Center \\ Moffet Field, CA. 94035 \\ 650-604-0159 \\ dpfletcher@mail.arc.nasa.gov \\ Rick Alena \\ NASA Ames Research Center \\ Moffet Field, CA. 94035 \\ 650-604-0262 \\ ralena@mail.arc.nasa.gov
}

Abstract-The computational infrastructure of the International Space Station (ISS) is a dynamic system that supports multiple vehicle subsystems such as Caution and Warning, Electrical Power Systems and Command and Data Handling (C\&DH), as well as scientific payloads of varying size and complexity. The dynamic nature of the ISS configuration coupled with the increased demand for payload support places a significant burden on the inherently resource constrained computational infrastructure of the ISS. Onboard system diagnostics applications are hosted on computers that are elements of the avionics network while ground-based diagnostic applications receive only a subset of available telemetry, down-linked via Sband communications.

In this paper we propose a scalable, out-of-band diagnostics architecture for ISS systems support that uses a read-only connection for C\&DH data acquisition, which provides a lower cost of deployment and maintenance (versus a higher criticality read/write connection). The diagnostics processing burden is off-loaded from the avionics network to elements of the on-board LAN that have a lower overall cost of operation and increased computational capacity. A superset of diagnostic data, richer in content than the configured telemetry, is made available to Advanced Diagnostic System (ADS) clients running on wireless handheld devices, affording the crew greater mobility for troubleshooting and providing improved insight into vehicle state. The superset of diagnostic data is made available to the ground in near real-time via an out-of band downlink, providing a high level of fidelity between vehicle state and test, training and operational facilities on the ground.

\footnotetext{
${ }^{1}$ U.S. Government work not protected by U.S. copyright

2 IEEEAC paper \#1013, Updated October 14, 2002
}

TABLE OF CONTENTS

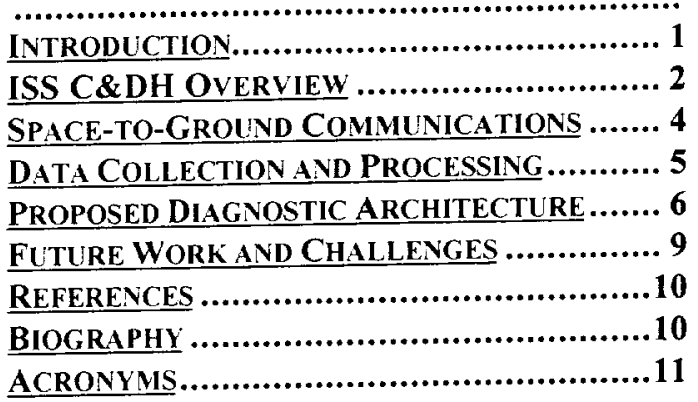

\section{INTRODUCTION}

As the backbone of the ISS computational infrastructure, the Command and Data Handling (C\&DH) system is composed of a number of specialized computers called Multiplexer/Demultiplexers (MDMs), arranged in a tiered hierarchy connected by MIL-STD $1553 \mathrm{~B}$ buses. The MDMs were built using state-of-the-art components at design time that were able to withstand the stringent environmental requirements of prolonged space flight such as radiation tolerance, heat dissipation characteristics and low power consumption. By today's standards, the ISS MDMs can be classified as resource constrained devices.

Due to the resource constraints of the Command and Control $(\mathrm{C} \& \mathrm{C}) \mathrm{MDMs}$ and the current space-to-ground communications architecture, the telemetry configured for downlink is only a subset of all diagnostic data available from the MDMs and 1553 buses in the tiered hierarchy. As the ISS grows in size, functionality and complexity, new Advanced Diagnostic Systems (ADSs) will be developed 
that give the crew greater insight into systems state and help flight controllers effectively manage ISS systems while minimizing or eliminating any increase in human resource expenditures. These ADSs will require a rich set of ondemand data without the intrusiveness of reconfiguring the telemetry tables of the C\&C MDMs.

Fault detection, isolation and recovery (FDIR) applications both onboard and on the ground rely on in-band data from the $\mathrm{C} \& D H$ system, acquired by configuring the telemetry tables of the C\&C MDMs through files uploaded via $\mathrm{S}$ band communications. Existing on-board FDIR components, which are active $\mathrm{C} \& D H$ elements, require a bidirectional (read/write) connection to the avionics system through a 1553 bus. The in-band, bi-directional nature of this connection puts the hardware and software components of the FDIR applications into a class of the highest criticality, making any expansion or upgrades to these components extremely expensive as well as imposing diagnostic constraints inherent to in-band management architectures.

This paper begins by presenting an overview of the Command and Data Handling system, the backbone of the ISS avionics and computational infrastructure. A similar background summary of the Space-to-Ground Communications System is presented. This background discussion serves as a basis for presenting the limitations of the existing diagnostic data dissemination architecture. We close by suggesting modifications and enhancements to the existing architecture that could yield greater insight into vehicle status both onboard and on the ground.

\section{ISS C\&DH OVERVIEW}

The U.S. on-orbit Segment (USOS) C\&DH System ${ }^{3}$ MDMs, interconnected by MIL-STD 1553B data buses, collect, process and distribute both data and commands to other MDMs acting as Bus Controllers (BCs) and other computational entities known as Remote Terminals (RTs). Not all RTs are MDMs, e.g. some are Portable Computer System (PCS) terminals that host applications enabling crew-members to send commands to the C\&C MDMs via a MIL-STD 1553 bus. Each bus has exactly one BC and some MDMs act as both BCs and RTs in the $\mathrm{C} \& D H$ tiered architecture.

The C\&DH system groups the computers and associated data buses into three tiers called the Control Tier, Local Tier and User Tier.

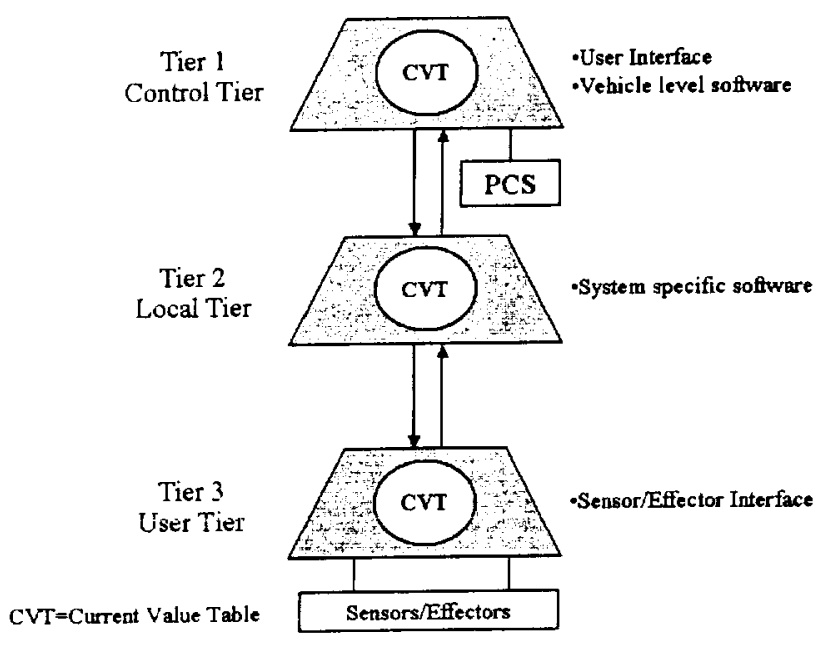

Figure 1: Conceptual View of the C\&DH Architecture.

MDMs and PCSs are major components of the ISS C\&DH system. Commands originate in the C\&C MDMs (Tier 1) pass through or are intercepted by Tier 2 (e.g. the Guidance, Navigation and Control) MDMs, move down through Tier 3 and then finally on to the sensors and effectors (temperature and pressure sensors, Remote Power Controllers, etc.). Conversely, telemetry from lower-tier devices traverses up the hierarchy to the C\&C MDMs. The crew and flight controllers interface with the ISS systems through Tier 1 only $^{4}$ via the PCS platform and S-band communications, respectively. The Current Value Table (CVT) is part of each MDMs memory and its data is used to populate PCS displays.

Generally, the Tier 1 MDMs are two-fault tolerant (three identical MDMs) and Tier 2 MDM and Tier 3 MDMs are one-fault tolerant (two identical MDMs). In the two-fault tolerant case, the active MDM is designated as hot, the backup is warm and the third is powered off or cold. The warm backup acts as an RT, processing data but not placing commands on the bus. In the one-fault tolerant case, one MDM is hot and the other is powered off . $^{5}$

The ISS C\&DH uses a cyclic data acquisition strategy, processing data at three different rates: $10 \mathrm{~Hz}, 1 \mathrm{~Hz}$ and 0.1 $\mathrm{Hz}$, with a processing rate of $80 \mathrm{~Hz}(12.5 \mathrm{~ms})$ internal to the MDM. These rate groups are referred to as a Major Cycle (10 second duration), Minor Frame (1 second duration), Processing Frame (100 millisecond duration) and Subframe, respectively. The BC broadcasts a Time Synchronization message at the beginning of each Processing Frame. MDMs that are RTs on the bus adjust their local time to that of the time received from the $\mathrm{BC}$ and compensate for the travel time down the bus. The different rate groups then share the bus through Time Division Multiplexing.

\section{MIL-STD I553B Protocol}

\footnotetext{
4 Some Tier 2 MDMs have a "pass-through" capability enabling commands to be sent to C\&C MDMs from buses between Tiers 2 and 3

${ }^{5}$ There are exceptions to this strategy, but they are not important here.
}

\footnotetext{
3 The Russian Onboard Complex Control System, the Russian Orbital Segment equivalent of the USOS C\&DH System, is not addressed in this work.
} 
The MIL-STD 1553B protocol is a deterministic, command-and-response protocol widely used in military and commercial aeronautics and aerospace applications with a nominal data rate of $1 \mathrm{Mbit} / \mathrm{s}$ for a 16-bit architecture. A single Bus Controller initiates command and data transfers on the bus, and Remote Terminals put data on the bus only when instructed to do so by the $\mathrm{BC}$. There can be up to 32 $\mathrm{RTs} / \mathrm{BC}$ on a bus (numbered 0 through 31 ), with one address reserved for the $B C$. However, the Station configuration allows only $31 \mathrm{RTs} / \mathrm{BC}$ on a bus with address 31 reserved as a broadcast address.

The 1553 protocol specifies fault tolerance of the bus and that fault tolerance is implemented using two separate channels for communication over twisted, shielded copper wires $^{6}$. If an error condition is detected on one of the channels, communication switches over to the other. In addition to the fault tolerance inherent to the 1553 protocol, the ISS C\&DH architecture provides fault tolerance by implementing redundant buses. If both channels on a bus are in failure mode, the MDM 1553 cards are configured to switch to the healthy bus. Hence, it takes four failed channels to disable bus communications.

The PCS hardware and software, which provides the crew interface to the C\&DH system, is configured as an RT on a Tier 1 or Tier 21553 bus and therefore is an integral part of the C\&DH system. Because of 1553 standards and MDM capacity, there are limitations on the number of PCSs that can be connected to each bus and to a single MDM. There can be a maximum of five PCSs on each bus, a maximum of eight PCSs connected directly under the C\&C MDM on the Control buses and a maximum of seven PCSs connected directly under the Payload MDM on the Payload Local buses [2]. These limitations must be taken into consideration to ensure that every PCS connection is recognized by the MDM.

It is important to note that the Bus Monitor mode and RTto-RT transfers, which are part of the 1553 standard, are not currently used by the ISS C\&DH system. In Bus Monitoring mode, the device is not an RT, therefore it never has commands directed to it by a BC nor will it ever put data onto the bus.

\section{C\&DH Hardware and Software}

For a variety of reasons relevant at design time, the ISS MDMs use an Intel 386SX microprocessor running at 32 $\mathrm{MHz}^{7}, 2 \mathrm{MB}$ of EEPROM, $8 \mathrm{MB}$ of RAM and certain MDMs have a Mass Storage Device (MSD) for data storage. The original MSDs had a spindle drive motor with read/write heads but have since been upgraded to solid-state components. Enhanced Space Station MDMs (ESSMDM) have High Rate Data Link (HRDL) cards with an optical interface that provide data access and retrieval to the

\footnotetext{
${ }^{6}$ An optical version of 1553 exists, but is not used onboard the ISS.

7 There are two types of MDMs on-board with minor differences: a Space Station MDM (SSMDM) and an Enhanced Space Station MDM (ESSMDM), where, generally, the Tier 1 and Tier 2 MDMs are ESSMDMs. The hardware statistics shown are for ESSMDMs.
}

associated MDMs MSD at data rates approximately 100 times faster than a 1553 network. The $386 \mathrm{SX}$ has a 16 -bit external data bus and a 32-bit internal data bus (hence a nominal $\mathrm{I} / \mathrm{O}$ data rate of $1 \mathrm{Mbit} / \mathrm{s}$ ). Each MDM host User Application Software (UAS), which differentiates the functionality of one MDM from another. For example, the C\&C MDMs host Command and Control Software (CCS) while the Guidance, Navigation and Control (GN\&C) MDMs host software specific to the GN\&C system. The UAS loads are characteristically real-time applications that process data at one or more of the previously mentioned rates in a real-time $\mathrm{ADA}$ Alsys run-time environment. A few MDMs are mounted externally to the ISS, making them susceptible to impact damage from micrometeorites in addition to being slightly more susceptible to radiationinduced Single Event Upsets (SEUs). At Assembly Complete, there will be 44 U.S. MDMs.

The PCS is the crew's primary command and control interface to the ISS core systems. The PCS computers are IBM Thinkpad 760XDs with a $166 \mathrm{MHz}$ Pentium processor; $64 \mathrm{MB}$ of RAM and a $3 \mathrm{~GB}$ hard drive, running the Solaris $\times 86$ operating system. The hardware platform is scheduled for upgrade to the IBM Thinkpad A3 lp (referred to as the Next Generation Laptop) with flight tests expected sometime in 2003 . These new notebook computers come equipped with a $1.7 \mathrm{GHz}$ Mobile Pentium 4 processor, 1 $\mathrm{GB}$ of RAM and a $60 \mathrm{~GB}$ hard drive, making them significantly more capable than the MDMs that control all of the subsystems on the Station.

The PCS laptop has a PCMCIA card that connects to a 1553 bus through a Portable Computer Receptacle (PCR) ${ }^{8}$. Each PCR provides power and data through the same connector, but only connects to one data bus. This bidirectional connection to the 1553 bus enables the PCS' primary capabilities of control and monitoring of core/payload systems and Caution and Warning (C\&W) notification and control.

The following table shows several operation transactions that take place between the MDMs and the PCS.

Table 1: Transactions and rates between MDMs and PCSs

\begin{tabular}{|l|l|c|}
\hline \multicolumn{1}{|c|}{ Description } & \multicolumn{1}{|c|}{ Direction } & $\begin{array}{c}\text { Transaction } \\
\text { Rate }\end{array}$ \\
\hline Display Data Transfer & To PCS & $10 \mathrm{~Hz}$ \\
\hline Standard Command & To PCS & $1 \mathrm{~Hz}$ \\
\hline Command from PCS & To C\&C MDM & $1 \mathrm{~Hz}$ \\
\hline Data Load & To PCS & $10 \mathrm{~Hz}$ \\
\hline File Dump & To C\&C MDM & $10 \mathrm{~Hz}$ \\
\hline
\end{tabular}

Note that commands sent to and from the PCS are limited to one command per second. Because of the 1553 bandwidth it takes approximately 3.5 minutes to transfer 1 $\mathrm{MB}$ of data from the MDM to a single $\mathrm{PCS}^{9}$, e.g. a 300

\footnotetext{
${ }^{8}$ There will be approximately 17 PCRs at Assembly Complete.

${ }^{9}$ The entire bandwidth of a 16-bit 1553 architecture is $1 \mathrm{Mbit} / \mathrm{s}$, but there are other RTs communicating on the same bus as the file transfer. The bus
} 
MB file would take approximately 17 hours to a single PCS, not including uplink time.

The PCS is susceptible to Single Event Effects (SEEs) that occur when a high-energy radiation particle impacts a component of the laptop. It is estimated that SEEs will occur as frequently as once every 9 hours. The effects of SEEs vary based on the type of event: Single Event Upsets (SEU) or Single Event Latch-up (SEL). An SEU is usually a bit-flip of non-critical memory and effects may go unnoticed. An SEL usually affects a critical area of memory and may require a power reset or system replacement and repair. Most SEEs are recoverable by rebooting the laptop. The occurrence of SEEs plays a critical role in the selection of hardware for both the PCS and the MDMs, both of which are categorized as Criticality $1^{10}$ components.

The Station Support Computer (SSC) provides crew support and utilization functions such as procedure viewing, inventory management and word processing and is the crew interface to the on-board Operations Local Area Network (OpsLAN). The OpsLAN consists of a laptop computer that acts as a central file server connected to a network of wireless access points and SSCs. The SSCs have the same hardware configuration as the PCS, but have a different software load running on a Windows NT operating system (the OS will be upgraded to Windows 2000 on the Next Generation Laptop). The SSC and OpsLAN were implemented to allow operations to be moved away from the PCS so that it is used primarily for control of ISS systems. A PCS can be converted to an SSC and vice versa simply by swapping out the hard drive. The OpsLAN and SSCs have no interfaces to vehicle commanding.

\section{SpaCe-to-Ground Communications}

The Communications and Tracking (C\&T) system is one of the major systems of the ISS and is divided into five subsystems: the Internal Audio Subsystem (IAS), the Sband Subsystem (S-band), the Ultrahigh Frequency (UHF) Subsystem, the Video Distribution Subsystem (VDS) and the Ku-Band Subsystem (Ku-band). Out of these five, our work is mainly concerned with the S-band and $\mathrm{Ku}$-band subsystems.

The S-band system transmits voice, commands, telemetry and files between the Station and ground. The IAS distributes audio onboard the Station to external interfaces. The VDS distributes video onboard the Station and to external interfaces, including the Ku-band for downlink.

The UHF Subsystem is used for Extra-Vehicular Activities (EVA) and proximity operations ", while the Ku-band Subsystem is used for payload data downlink and video and file two-way transfer. NASA is studying plans to add the

\footnotetext{
is not dedicated to the file transfer operation.

${ }_{10}$ A component is generally classified as Criticality 1 if failure modes could result in serious injury or loss of life (flight or ground personnel), or loss of vehicle.

11 Proximity operations occur when the Shuttle is approaching or departing the Station.
}

capability to transfer commands and data between the ground and the USOS over Ku-band as a backup to the Sband. The following figure shows the command path from the ground to the Station via S-band.

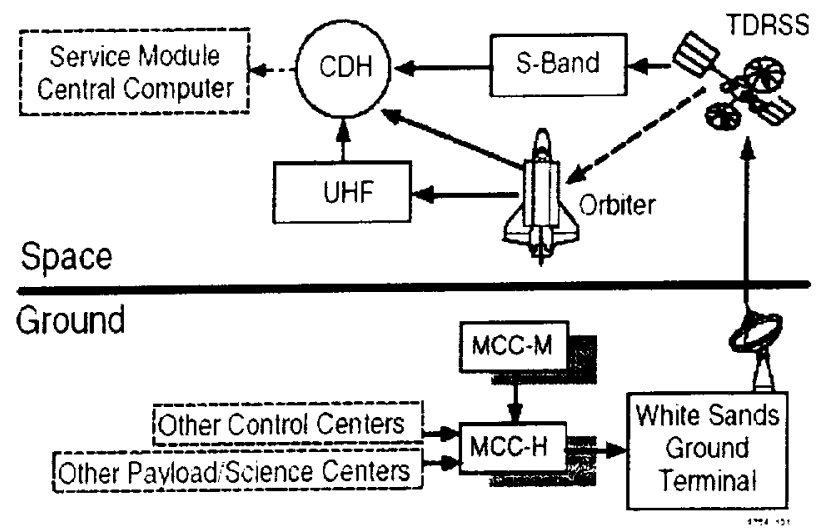

Figure 2: USOS Command Paths ${ }^{12}$

The ISS C\&T system uses the NASA Tracking and Data Relay Satellite (TDRS) System for RF communications between the Station and ground. The TDRS System satellites are in geosynchronous orbit (approximately 36,000 $\mathrm{km}$ ) while the Station's mean orbital altitude is approximately $370 \mathrm{~km}$ with a period of about 90 minutes. The TDRS system is a line-of-sight system where the minimum orbital altitude for $100 \%$ view is approximately $1200 \mathrm{~km}$. The low orbital altitude of the Station relative to the geosynchronous orbit of the TDRS system results in Loss-of-Signal (LOS) events lasting approximately 10 minutes per orbit. The period of LOS is referred to as the Zone of Exclusion (ZOE). One of the major purposes of the previously mentioned MSD attached to certain ESSMDMs is to store data that cannot be downlinked during the ZOE. When the ISS regains TDRS signal, the data saved on the MSD is dumped to the ground via either S-band or Ku-band.

\section{S-band System}

The S-band System is the communication system used for primary Command and Control of the ISS. It transports commands originating from the Mission Control CenterHouston (MCC-H) and the Payload Operations Integration Complex (POIC) to the ISS and transports USOS System and critical telemetry from the ISS to the Tracking and Data Relay Satellite System (TDRSS) and ultimately to the MCC-H and POIC. Telemetry data can be "real-time" or recorded telemetry data. The S-band is also used for twoway audio as well as file transfer between the ground and the C\&DH system. Files are sent by flight controllers to an MDM to change configurations, limits, etc. and make their way through to the C\&DH system via the 1553 C\&T Control Bus connected to the S-band system's Baseband

\footnotetext{
${ }^{12}$ MCC-H/M = Mission Control Center-Houston/Moscow. The Service Module Central Computer is in the Russian Segment
} 
Signal Processor, where the data is decrypted before going onto the bus.

The highest data rate at which the S-band system transmits and receives is $192 \mathrm{kbps}$ on the return link (the communications path from the ISS to TDRSS and then to $\mathrm{MCC}-\mathrm{H})$ and $72 \mathrm{kbps}$ on the forward link.

The S-band system is a two-string system (single fault tolerant) with no connection between the two strings. The backup system uses a lower data rate of $6 \mathrm{Kbits} / \mathrm{s}$ on the uplink and $12 \mathrm{Kbits} / \mathrm{s}$ on the downlink, with no support for audio.

\section{Ku-band System}

The Ku-band System's main purpose is to provide a High Data Rate return link (from Station to the ground) for realtime and recorded payload data, video (real-time and recorded), and ISS systems telemetry recorded during the ZOE. There is also a forward link capability to support the two-way transfer of files and video teleconferencing in support of the OpsLAN. The ISS Ku-band system nominally sends up to $150 \mathrm{Mbits} / \mathrm{s}$ of serial data to the ground using up to 12 different channels. System overhead is about $6.8 \mathrm{Mbits} / \mathrm{s}$, leaving about $143 \mathrm{Mbits} / \mathrm{s}$ of usable capacity. Up to 4 of the 12 channels can contain video images from the VDS. Up to 8 of the channels are reserved for payload data and two of the payload data channels are shared between transmitting recorded telemetry and recorded payload data. The Ku-band forward link is at a lower data rate than the return link. Dumping the $\mathrm{ZOE}$ telemetry through $\mathrm{Ku}$-band takes minutes as compared to hours via Sband.

Although the $\mathrm{Ku}$-band system provides a greater data rate than the S-band, it is less robust. The S-band system has two-strings while the Ku-band system is single-string (zero fault-tolerant). Furthermore, structural blockage from the ISS itself greatly impacts the downlink communication availability with only approximately 70 percent coverage per orbit, on average, at Assembly Complete. This greater ZOE for Ku-band has a negative impact on available throughput, but throughput is still significantly greater than that of $\mathrm{S}$ band.

The Ku-band system connects to the C\&DH system through an Automated Payload Switch (APS), connected to the C\&C MDMs via the HRDL card on the C\&DH side and via a TAXI $^{13}$ interface to the High Rate Frame Multiplexer (HRFM) on the Ku-band side. The recorded ISS telemetry goes through the APS to the HRFM and then on to the ground.

The Ku-band system connects to the OpsLAN through an Orbital Communication Adapter (OCA) Router, providing Internet Protocol (IP) network connectivity between the ground and the OpsLAN. The OCA Router is a card that slides into a laptop computer, providing full-duplex RF-to -

\footnotetext{
${ }^{13}$ A point-to-point optical interface with a data rate of $100 \mathrm{Mbps}$
}

Ethernet communications adaptation. The Cisco IP SoftPhone ${ }^{T M}$ application has been successfully deployed on the OpsLAN, out-of-the-box, through the OCA Router with only a minor modification to a TCP configuration parameter to deal with the latency introduced by a DOMSAT link (not shown in Figure 2) between MCC-H and White Sands, NM.

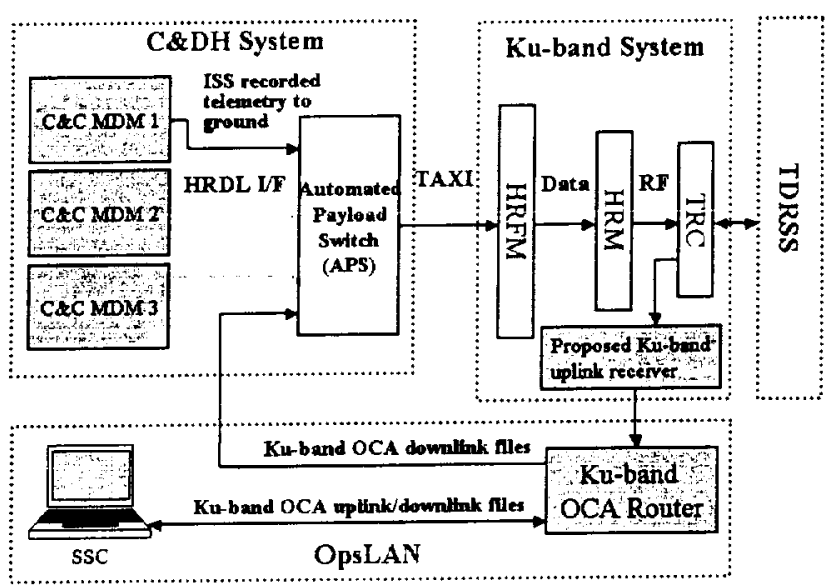

Figure 3: Ku-band Overview Schematic

\section{Data Collection and Processing}

Data is collected from sensors, effectors and firmware controllers and is stored in the MDMs CVT. This data is then passed upwards in the C\&DH tiered hierarchy until it reaches the C\&C MDM. The C\&C MDM CVT contains the composite of data generated from all ISS systems and is made available to the user, regardless of the origin of the data. From the C\&C CVT, the data is distributed to the crew via the PCS and to the ground through the Communications and Tracking (C\&T) System.

The ISS generates several times more diagnostic data than can be downlinked to the ground through the S-band communications system (the only path currently available for downlinking real-time telemetry). Therefore, the ground has to choose a subset of the data stored in the CVT to be downlinked as cyclic telemetry. A new configuration file can be uploaded to the C\&C MDM to select which parameters are downloaded according to current needs. However, when ground controllers are engaged in fault detection, isolation and recovery (FDIR) efforts, deciding which parameters are relevant to root-cause analysis is an error-prone task at best and can be nearly impossible due to the complexity of the ISS systems. Data dumps are used to downlink selected areas of an MDM's memory that are not included in cyclic telemetry. Ground personnel use these dumps to look at additional data necessary for failure investigation. Data dumps can only be initiated from the ground, since the PCS does not have the ability to determine what memory addresses in the MDM to dump. Data can only be dumped from one MDM at a time.

The MDMs do not verify the validity of the data stored in the CVT, resulting in the possibility of there being stale 
data in the CVT. There is no indication that the affected data in the CVT is not being updated. This is commonly referred to as "the stale data problem". An example of a situation that results in stale data being displayed on a PCS is an $\mathrm{I} / \mathrm{O}$ card failure, a bus failure or a sensor failure.

The C\&DH system has two types of automated FDIR software. The first type, called Bus FDIR, is a common set of software located in the memory of all MDMs that act as BCs. The Bus FDIR software automatically detects three things: channel failures, loss of bus communication with an $\mathrm{RT}$, and loss of bus communication from the BC. Channel failures result in an automatic channel switchover, which makes the redundant channel active. If the second channel fails, then the bus is considered failed. The automatic switchover can be enabled or disabled by the crew or MCCH.

The second type of automated C\&DH FDIR software is referred to as Remote Terminal (RT) FDIR. This type of FDIR handles MDM failures such as loss of communication or total loss of the MDM. Generally, RT FDIR is dependent upon the tier of the MDM (characterized by its redundancy). For Tier 1 and Tier 2 MDMs, the RT FDIR determines the type of failure and switches to a redundant MDM if appropriate. Due to the complex hardware/software redundancy of Tier 3, the Tier 3 RT FDIR typically varies by MDM.

The S-band flight software provides FDIR for the S-band Subsystem by evaluating key status parameters from the elements that comprise the subsystem. When a critical failure is detected, S-band FDIR tells the Caution and Warning system to issue a Caution alarm. There are two flight software applications for S-band recovery: S-band String Recovery and S-band Extended Loss of Communication Recovery.

Currently, all ISS system diagnostics are performed using an in-band data acquisition and analysis strategy, described thus far, where the critical system under diagnosis is used to deliver and in the case of the PCS, display, diagnostic data. The current Station critical systems management framework closely resembles that of the in-band management strategy of the Simple Network Management Protocol (SNMP) where the cyclic telemetry configured for downlink from the CVT is analogous to an SNMP Management Information Base (MIB), the PCS and ground-based diagnostic applications analogous to SNMP Clients and the MDM UAS software analogous to the SNMP Agent. Also analogous to the SNMP in-band management architecture is the fact that the network being managed (here, the combined 1553 network and S-band link) is used to transport the management data. All three components: the Agent, the Client and the network must be functional for in-band management to work effectively.

Out-of-band management requires neither a functioning Agent nor that the network under management be operating in order to work.

\section{Proposed Diagnostic Architecture}

The first modification we suggest is that the Ku-band communication system be implemented as a backup to the critical S-band system, enabling the downlink of cyclic telemetry in the event of S-band communications failure. Perhaps the greatest obstacle to implementing this modification, however, is that commanding from the ground would require a two-way physical connection onboard between the C\&DH system, designed and tested to the standard of highest criticality, and the Ku-band system, designed and tested to a lower standard of criticality. This mismatch in design standards would have one sending commands to a Criticality 1 entity through a system having a lower criticality rating. However, with better insight into the state of the vehicle through Advanced Diagnostic Systems, the need for commanding over Ku-band could be minimized. For example, with enhanced insight into the system, the ground may be able to successfully perform a root cause analysis using only one data dump rather than several.

Regardless of whether the Ku-band system is implemented as a backup for S-band, ISS Program budget considerations require that flight controllers do more with less, and their workload will only increase as the ISS nears Assembly Complete, fulfilling its primary role as a platform for scientific research. As the ISS grows in size and complexity, flight controllers and other ground support personnel would benefit from having a richer set of telemetry at their disposal.

As noted above, a one-way connection already exists between the C\&DH and Ku-band systems for downloading ZOE files from the MSD via the APS. A second possible interface exists between the C\&DH and Ku-band systems through the OpsLAN. We suggest using this as an entry point for a one-way, read-only connection to the 1553 network using an SSC platform hosting a card configured as a 1553 Bus Monitor. Since the 1553 protocol guarantees that a Bus Monitor cannot put data onto the bus, the connection between the Criticality $1 \mathrm{C} \& D H$ system and the lower criticality OpsLAN will not violate safety specifications.

Due to the nature of the tiered hierarchy of the C\&DH system, the vast majority of data traversing the system over the 1553 network can be acquired by connecting as a Bus Monitor to four high-level buses:

1. CB-CT: the Control Bus used by Communications and Tracking for telemetry downlink and commanding,

2. CB-INT: the Control Bus used by MDMs controlling internal subsystems such as the Electrical Power System (EPS), Environmental Control and Life Support System the Thermal Control System (TCS), as well as some capabilities to support docking and the Caution and Warning System,

3. CB-EXT: the Control Bus used by MDMs monitoring data on the various truss segments and controlling some EPS and TCS functions, and 
4. CB-GNC: the Control Bus used by the Guidance, Navigation and Control MDM.

We know that the current $C \& D H$ architecture allows for a maximum of eight PCSs connected directly under the C\&C MDM on the Control Buses. Since our proposed connections will be Bus Monitors and not RTs that must be serviced by an MDM, implementing the four connections mentioned above will not place additional burden on the MDMs thereby leaving at least another four connections open for PCS units.

\section{Diagnostic Data Server Architecture}

We have implemented a prototype of our proposed system in the Intelligent Mobile Technologies (IMT) lab at NASA Ames Research Center. This prototype system, called the Diagnostic Data Server (DDS), was developed using 1553 data collected from test runs at the International Space Station Systems Laboratory (ISIL) at Johnson Space Center. The ISIL is the highest fidelity test and integration facility available for the ISS. The Hardware/Software Integration (H/SI) group at ISIL collected and archived the 1553 data on the four Control Buses mentioned above during test runs and made that data available to us in the IMT lab.

The archived 1553 files are played back using the SBS PASS $^{\top M}$ product on a host computer equivalent to the SSC. This data source computer uses the PASS software to drive the archived data onto a 1553 bus, maintaining the integrity of the time deltas between 1553 messages, for consumption by a data sink host with a 1553 card configured as a Bus Monitor.

There is one Bus Monitor host for each of the four Control Buses. Each Bus Monitor host runs two processes: 1) a Data Collection Engine (DCE), which is responsible for reading the messages from the 1553 card using the interface library supplied by the vendor, and, 2) a Decommutator, which is responsible for extracting parameters called Program Unique Identifiers (PUIs) ${ }^{1 / 4}$ from the 1553 messages. These PUIs are derived data values consumed by Artificial Intelligence-based ADS applications for prediction of ISS systems behavior and fault detection, isolation and recovery.

The PUI values produced by the four Decommutators are aggregated onto a single server residing on the OpsLAN where the data is archived and distributed to multiple clients using a publish/subscribe architecture. The Decommutators send messages containing a time stamp, name, value tuple to the server using TCP socket connections. Data from all three rate groups: $10 \mathrm{~Hz}, 1 \mathrm{~Hz}$ and $0.1 \mathrm{~Hz}$, are decommutated and checked to see if there is a change from the last recorded value. Only changed data is sent to the server. The decommutator sends a time-out message to the server for a PUI if the value has not changed for some configurable time frame, thereby addressing the "stale data

\footnotetext{
14 All parameters associated with ISS systems have an associated Program Unique Identifier.
}

problem". The PUIs the user wants decommutated are listed in an XML configuration file, built by an external program that interacts with the Mission Build Facility's (MBF) Standard Output (StdOut) Definition Database. The MBF StdOut Database defines each characteristic of all PUIs defined for the ISS, such as the rate group a PUI belongs to, the 1553 message it is contained in, the word offset and bit offsets into the 1553 message, it's data type, and more, including instructions on unit conversion if the PUI is an analogue signal. Decommutators for different buses are differentiated only by their configuration files. 
The Data Aggregation Server platform hosts an instance of the open-source, freely available JBOSS implementation of the Java 2 Enterprise Edition (J2EE) Specification. The Java Messaging Service, defined by the J2EE spec and implemented in JBOSS, is used for the publish/subscribe infrastructure and the Tomcat servlet container is used for servicing web clients. The Decommutators publish the PUI tuples to the Data Aggregation Server, and ADS clients can subscribe to individual PUIs or to all PUIs associated with a given subsystem such as C\&DH, EPS, TCS or ECLSS.

The first ADS client prototype we developed as a DDS
JPEG image is written into a repository using the Java Advanced Imaging class libraries. These images of system state are then served out to hand-held clients connected to the "OpsLAN" in the IMT lab through a RangeLAN2 wireless network, the same type of wireless network used onboard. The image repository design approach was chosen in order to off-load processing responsibilities from the hand-held clients in consideration of battery life. All the client platform has to know how to do is display a JPEG image since all processing is done on the server side. The trade-off for using this approach is increased bandwidth utilization across the wireless network for transporting

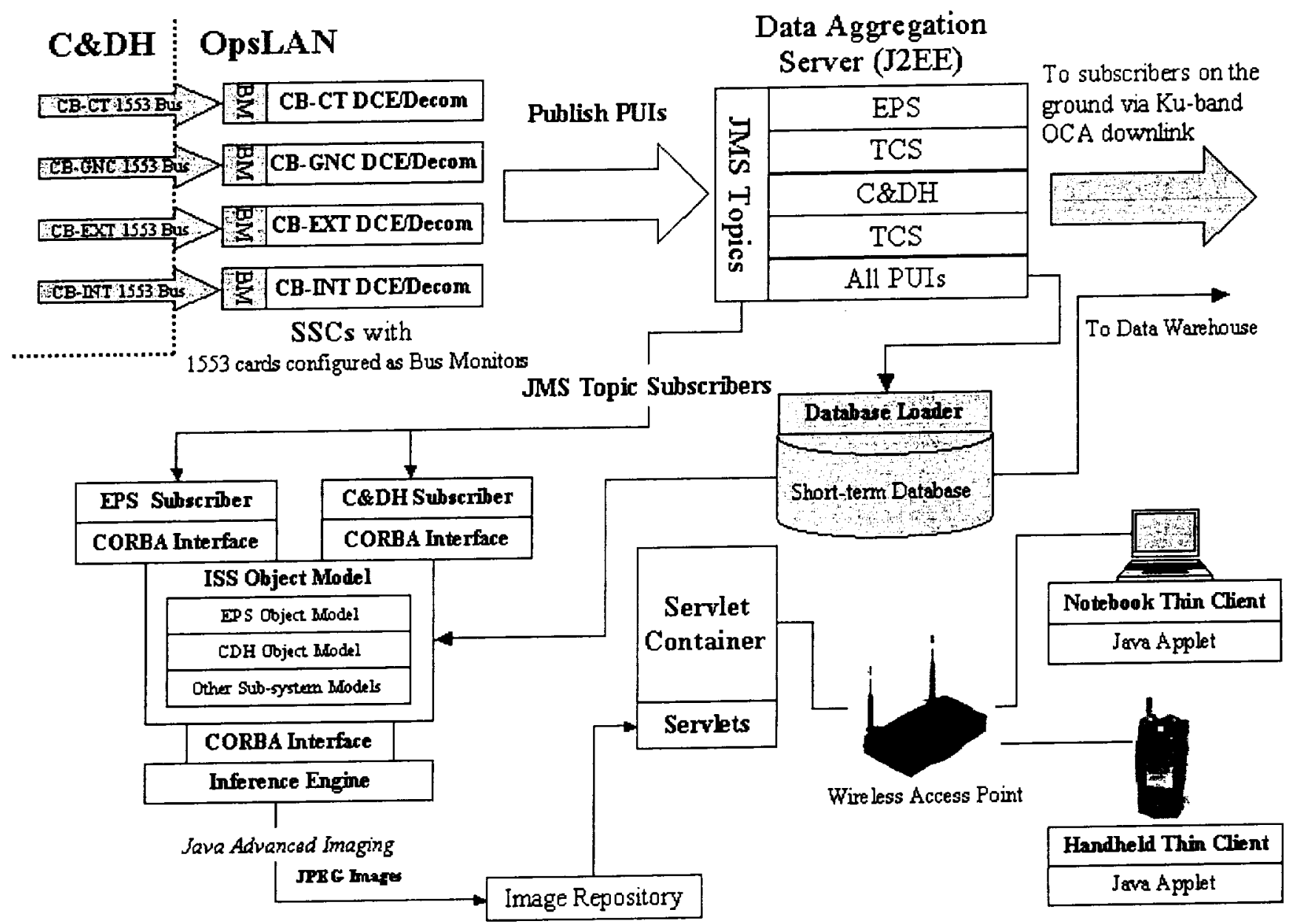

Figure 4: Diagnostic Data Server Architecture Schematic

client was an Object Model of the ISS, populated with the PUI values obtained by subscribing to the subsystem "topics" available from the Aggregation Server. Through the DDS, the Object Model has access to all 1553 data on the four Control Buses, a superset of what would be available via cyclic telemetry downlink. A rule-based Inference Engine determines high-level vehicle state by interacting with the Object Model, creates an image of the current vehicle state in memory, and writes the image out to disk as a JPEG image when there is a change in system state. The images as opposed to data values.

The Data Aggregation Server is a part of the OpsLAN and is therefore connected to the ground through the OCA Router and the Ku-band system. The fact that the Cisco SoftPhone ${ }^{\circledR}$ successfully establishes a TCP/IP connection to the ground suggests that client ADS applications on the ground can subscribe to the same "topics" and superset of data to which the onboard clients subscribe since the Messaging Service on the Aggregation Server is built on 
TCP. However, the Cisco SoftPhone ${ }^{\circledR}$ adjustment to the TCP stack parameters only address the latency associated with establishing a connection. This configuration change does not account for what would happen to a TCP connection when the Station goes in and out of the ZOE.

The PUI database is used for short-term storage of changeonly data and could be accessed by ground-based applications to acquire data recorded during the ZOE. Four fully loaded 1553 buses produce $0.5 \mathrm{MB} / \mathrm{s}$ or approximately 43 GB of data per day. Storing change-only data reduces the volume, but further work must be done to derive a reliable percent reduction estimate. Since the short-term data warehouse will likely reside on an SSC in the near-term, understanding the actual volume of data stored will be important since the Next-Generation Laptops to be used for SSC upgrades have $60 \mathrm{~GB}$ hard drives.

A prototype data warehouse is currently under development in the IMT lab where ADS developers use data mining techniques for algorithm and application development. The short-term database in our prototype architecture is implemented using the open-source MySQL Database. The current release of MySQL does not support transactions ${ }^{15}$, so we use PostgreSQL, an alternative open-source database that does support transactions, for the prototype data warehouse.

Using tools under development in the IMT lab, ADS developers use the data warehouse to create and playback fault-scenarios through a web portal provided as an interface to the Integrated Vehicle Health Management (IVHM) test bed in the lab. The IVHM test bed is a work-in-progress that aggregates ISS data sources from disparate locations, such as test data sets from the ISIL and historic ISS telemetry from the Operational Data Reduction Complex (ODRC), providing a unified interface to multiple data sources for ADS model developers.

The mobile client in our prototype architecture is hosted on the Compaq iPaq 3850 running the Microsoft PocketPC operating system, a Pocket Internet Explorer web browser, and the Jeode ${ }^{\mathrm{TM}}$ Java Embedded Virtual Machine from Insignia Solutions, Inc. The prototype ADS client receives updated status images of the C\&DH system by interacting with a servlet on the Data Aggregation Server at a configurable polling rate. Currently, the crew's decision support tools are hosted on the PCS platform, which must be connected to the 1553 network at fixed locations. Through the use of the wireless network, our prototype DDS architecture gives ADS clients untethered access to diagnostic data anywhere within range of the OpsLAN wireless access point.

\section{Summary-Diagnostic Data Server}

The diagnostic data made available to the ADS clients through the DDS is a superset of the C\&DH cyclic

\footnotetext{
15 Transactions, triggers and stored procedures are planned for later releases of MySQL
}

telemetry, acquired without placing additional burden on the MDMs since the 1553 cards in the DCE/Decom host computers are configured as Bus Monitors, not as RTs. The diagnostic data is acquired and disseminated out-of-band from the C\&DH and S-band systems, and therefore does not rely on the 1553 network or the S-band link to be functioning in order to work. In the event of failure of a 1553 bus, the DCE processes can detect bus the failure and the pertinent diagnostic data is disseminated to ADS clients via a separate network (OpsLAN and $\mathrm{Ku}$-band). The number of Bus Monitors available to the DDS is limited by the 1553 protocol, but is scalable in the sense that multiple clients can be served without adding connections to the 1553 network. The DDS implementation uses open-source software wherever possible and uses Commercial Off-TheShelf (COTS) hardware. Due to the nature of its design, the DDS will be less expensive to flight qualify and upgrade than Criticality 1 Station diagnostics components.

\section{Future Work and Challenges}

The IMT lab is working to bring the prototype DDS up to flight-quality standards on a track of formal testing, validation and verification for deployment as a flight experiment to support Advanced Diagnostic and Decision Support Systems both onboard and on the ground at MCC$\mathrm{H}$. Our intention is to use the Next-Generation Laptop (NGL) SSC upgrade as the onboard deployment platform for the DDS flight experiment. NASA has specified Microsoft Windows2000 as the operating system for the SSC platform. Since the DCE/Decom processes interact with a real-time system, these processes posses real-time characteristics with deadlines to meet at the $10 \mathrm{~Hz}, 1 \mathrm{~Hz}$ and $0.1 \mathrm{~Hz}$ data rates. It is likely that in order to be effective at processing all 1553 messages on a bus, the DCE/Decom will have to be developed as a real-time application hosted on a real-time operating system. Further load testing is required to determine the suitability of the SSC upgrade as a Data Aggregation Server platform for DDS.

Using a single Data Aggregation Server in the DDS architecture creates a single-point of failure and does not easily scale "up" to handle additional loads introduced by payloads and C\&DH expansion through Assembly Complete. The weight and size of computational components contributes significantly to their cost of deployment since they must be transported to the ISS as payload on the Shuttle. The group in the IMT lab has evaluated several different COTS rack-mounted servers running the Windows 2000 and Linux operating systems for suitability as OpsLAN server elements. Aside from radiation tolerance, operational characteristics such as physical dimensions (the server needs to fit in an ISS payload rack, not a typical $42 \mathrm{U}$ rack enclosure), power consumption, heat dissipation and fan noise, are important evaluation criteria. Even though some rack-mounted servers can be re-packaged to fit into a payload rack, we found that the power consumption, heat dissipation and noise characteristics made the COTS rack-mounted servers unsuitable for deployment onboard. Our next step is to 
identify and evaluate some server candidates from the new Blade Server technology. Server blades are generally designed for high-density deployment using ultra-low voltage processors and could provide a relatively inexpensive way to add additional computational capacity to the OpsLAN, as well as add scalability and fault-tolerance to the DDS. Since the blades would be a part of the OpsLAN, and not meant to do double-duty as Criticality 1 systems (as is the PCS/SSC hardware), they could be less expensively qualified to a lower-radiation tolerance than C\&DH components.

Another area of further study regards maintaining TCP/IP connectivity between the Station and ground during the ZOE. A group associated with NASA JPL ${ }^{16}$ has produced the Space Communications Protocol Standards (SCPS), a suite of standard data handling protocols that specifically address the problems associated with IP network connectivity in the space environment. Of particular interest to our work with DDS are the SCPS-FP, SCPS-TP and SCPS-SC protocols, analogous to the common FTP, TCP and IPSEC protocols, respectively. The SCP Standards are being progressed toward full ISO standards through the work of the international Consultative Committee for Space Data Systems (CCSDS) ${ }^{17}$. Using these protocols between the ISS and ground could enable the use of COTS applications on the OpsLAN without modification for the ZOE, since the underlying protocol makes the discontinuity transparent to applications at higher layers of the ISO stack.

The ISS OpsLAN uses Proxim RangelaN2 ${ }^{\text {TM }}$ products, based on the OpenAir standard, for wireless connectivity. The OpenAir standard is used for the wireless LAN (WLAN) because it was found less susceptible to multipath effects than $802.11 \mathrm{~b}$ in the "tin can" environment of the ISS. The RangeLAN2 ${ }^{\text {TM }}$ products are Frequency Hopping Spread Spectrum (FHSS) radios. Though it is known that the Orthogonal Frequency Division Multiplexing (OFDM) used in 802.11a WLANs reduces crosstalk compared to FHSS, it is unclear whether OFDM radio components would be as well behaved as the OpenAir FHSS components in an environment dominated by multipath effects. An upgrade of the RangeLAN2 ${ }^{\text {TM }}$ WLAN to 802.11 a would provide an approximately four-fold increase in bandwidth for the wireless portion of the OpsLAN. Further study is needed in this area.

The true utility of the DDS lies in its ability to serve data to client applications that make the tasks of crew and ground personnel easier, enabling them to "do more with less". Advanced Diagnostic Systems are being developed to give the crew and ground greater insight into an increasingly complex system. Prototype ADSs, initially target for use by flight controllers at MCC-H, are under development for the ISS C\&DH and Electrical Power Systems by the Livingstone Group at Ames Research Center and the Institute for the Study of Learning and Expertise (ISLE) ${ }^{18}$ in

\footnotetext{
${ }^{16}$ http $/ /$ www. scps.org/

17 The ISS formats telemetry from the CVT in CCSDS packets for downlink over S-band as well as other data downlinked over Ku-band.

${ }_{18}$ ISLE is a non-profit organization dedicated to research and education
}

Palo Alto, CA., respectively. Further work is needed to refine the underlying models of the ADS prototypes under development, to better understand the interactions and complex relationships between the ISS subsystems, to represent and communicate those complex interactions amongst ADS subsystem models and to define new ADS efforts to support the goals of the ISS Program.

\section{REFERENCES}

[1] Mission Operations Directorate Space Flight Training Division, International Space Station Familiarization, July 31, 1998.

[2] Mission Operations Directorate Space Flight Training Division, Command and Data Handling Training Manud, June 2000.

[3] Mission Operaions Directorate, User's Guide for the Portable Computer System (PCS), Revision C, May 24, 2001.

[4] Intemational Space Station Program, "Software Interface Control Document Station Management and Control to Intemational Space Station Book 2, General Software Interface Requirements", Revision D, April 1, 2000.

\section{BIOGRAPHY}

Daryl Fletcher received his B.S. degree in Applied Mathematics in 1993 and an M.S. degree in Engineering in 1995 from the University of Colorado at Boulder. His background is in systems development for aviation meteorology and development of Network Management Systems for data and voice networks. In graduate school, his research interest was in advanced numerical methods for the solution of stochastic partial differential equations. He has extended that research interest to the application of stochastic and fractal modeling for IP network traffic management and modeling the behavior of complex systems. Prior to joining SAIC, he was a software developer at the National Center for Atmospheric Research in Boulder, CO. and a consultant to Lucent Technologies and Level(3) Communications.

in machine learning, artificial intelligence and cognitive science. ISLE Home Page 


\section{ACRONYMS}

$\begin{array}{ll}\text { ADS } & \text { Advanced Diagnostic System } \\ \text { APS } & \text { Automated Payload Switch } \\ \text { BC } & \text { Bus Controller } \\ \text { CB } & \text { Control Bus } \\ \text { CCS } & \text { Command and Control Software } \\ \text { C\&C } & \text { Command and Control } \\ \text { C\&DH } & \text { Command and Data Handling } \\ \text { C\&T } & \text { Communications and Tracking } \\ \text { CVT } & \text { Current Value Table } \\ \text { DDS } & \text { Diagnostic Data Server } \\ \text { ECLSS } & \text { Environmental Control and Life Support System } \\ \text { EPS } & \text { Electrical Power System } \\ \text { FDIR } & \text { Fault Detection, Isolation and Recovery } \\ \text { GNC } & \text { Guidance, Navigation and Control } \\ \text { HRDL } & \text { High Rate Data Link } \\ \text { HRFM } & \text { High Rate Frame Multiplexer } \\ \text { HRM } & \text { High Rate Modem } \\ \text { IMT } & \text { Intelligent Mobile Technologies } \\ \text { ISIL } & \text { ISS Systems Integration Lab } \\ \text { ISS } & \text { International Space Station } \\ \text { LOS } & \text { Loss of Signal } \\ \text { MBF } & \text { Mission Build Facility } \\ \text { MCC-H } & \text { Mission Control Center-Houston } \\ \text { MCC-M Mission Control Center-Moscow } \\ \text { MDM } & \text { Multiplexer/Demultiplexer } \\ \text { MSD } & \text { Mass Storage Device } \\ \text { OCA } & \text { Orbital Communication Adapter } \\ \text { PCS } & \text { Portable Computer System } \\ \text { POIC } & \text { Payload Operations Integration Complex } \\ \text { PUI } & \text { Program Unique Identifier } \\ \text { RT } & \text { Remote Terminal } \\ \text { SCPS } & \text { Space Communications Protocol Standards } \\ \text { SSC } & \text { Station Support Computer } \\ \text { StdOut } & \text { Standard Output Definition Database } \\ \text { TCS } & \text { Thermal Control System } \\ \text { TDRSS } & \text { Tracking and Data Relay Satellite System } \\ \text { TRC } & \text { Transmitter/Receiver Controller } \\ \text { UAS } & \text { User Application Software } \\ \text { ZOE } & \text { Zone of Exclusion } \\ & \end{array}$


